\title{
3.8dB Noise Figure in Bulk Semiconductor Optical Amplifier
}

\author{
K. Carney*, R. Lennox**, R. Maldonado-Basilio*, S. Philippe*, F. Surre***, L. Bradley** and P. \\ Landais* \\ * The Rince Institute, School of Engineering, Dublin City University, Glasnevin, Dublin 9, Ireland \\ ** School of Physics, Trinity College Dublin, Dublin 2, Ireland \\ *** School of Engineering and Mathematical Sciences, City University London, London EC1V 0HB, United Kingdom
}

\section{INTRODUCTION}

Semiconductor Optical Amplifier (SOA) has been widely researched and its interest for optical networks has been demonstrated to perform different functions up to $320 \mathrm{~Gb} / \mathrm{s}$, such as power booster, pre-amplification, wavelength conversion [1]. However, commercial devices are limited by a significantly higher Noise figure (NF) than Erbium Doped Fiber Amplifier.

Many techniques have been developed to reduce the NF. The main one is the Gain-Clamped SOA (GC-SOA) [2], where Distributed Bragg Reflectors mirrors at both facets create a laser cavity. The lasing mode clamps the carrier density, which reduces the carrier density fluctuation. The clamping can also be realised by vertical cavity. These two techniques present the same drawbacks: in order to achieve a given gain, it is necessary to have a larger population inversion, which means the GC-SOA must be driven by a larger current than that of a conventional SOA. For similar bias current, gain-clamped SOAs will present a lower gain and a higher NF due to a lower carrier density.

In this paper, a different approach is proposed. The reduction in NF is obtained by carefully engineering the carrier density inside the SOA. To this aim, the metallic contact is split into three sections driven by independent currents. A numerical model is derived to confirm the NF reduction. Then, devices are fabricated and their NF is measured after increasing the resistance inter-contact using focused ion beam (FIB) technique.

\section{MODEL}

\section{A. Carrier Density Distribution}

The model used to characterize the NCSOA is based on [3]-[4]. The device active layer is longitudinally divided in sections. In each section, the carrier density is considered being constant and, as a consequence, also is the gain. The carrier density is calculated by solving the $\mathrm{CW}$ rate equation in each section:

$$
\frac{\mathrm{dN}_{\mathrm{m}}}{\mathrm{dt}}=\frac{\mathrm{I}_{\mathrm{m}}}{\mathrm{qV}}-\mathrm{R}\left(\mathrm{N}_{\mathrm{m}}\right)-\mathrm{v}_{\mathrm{g}}\left[\mathrm{g}\left(\omega_{\mathrm{sig}}, \mathrm{N}_{\mathrm{m}}\right) \cdot \mathrm{S}_{\mathrm{m}, \text { sig }}+\mathrm{g}\left(\omega_{\mathrm{spon}}, \mathrm{N}_{\mathrm{m}}\right) \cdot \mathrm{S}_{\mathrm{m}, \mathrm{spon}}\right]
$$

where $N_{m}$ is the density of carriers in the $\mathrm{m}^{\text {th }}$ section, $I$ is the bias current, $q$ is the carrier charge, $V$ is the volume of the active region, $v_{g}$ is the group velocity, $R\left(N_{m}\right)$ represents the recombination rates equal to $\mathrm{AN}_{\mathrm{m}}+\mathrm{BN}_{\mathrm{m}}{ }^{2}+\mathrm{CN}_{\mathrm{m}}{ }^{3}$, where $\mathrm{A}$ is the non radiative recombination coefficient, $\mathrm{B}$ the radiative recombination coefficient and C the Auger recombination coefficient, $g$ is the material gain coefficient. Its expression can be found in [5]. $S_{m, s i g}$ and $S_{m, \text { spon }}$ are the photon density for the injected signal and spontaneous emission respectively. How to calculate them can be found in [4].

\section{B. Noise Figure}

The noise figure for one section can be expressed as:

$$
\mathrm{nf}=\frac{2 \mathrm{n}_{\mathrm{sp}}(\mathrm{g}-1)}{\mathrm{g}}+\frac{1}{\mathrm{~g}}
$$

where $g$ is the single pass chip gain and $n_{s p}$ is the population inversion factor.

The total noise figure of the SOA can be estimated by using Friis' formula for cascading amplifiers:

$$
\mathrm{nf}_{\text {total }}=\mathrm{nf}_{1}+\frac{\mathrm{nf}_{2}-1}{\mathrm{~g}_{1}}+\ldots+\frac{\mathrm{nf}_{\mathrm{m}}-1}{\mathrm{~g}_{1} \ldots \mathrm{g}_{\mathrm{m}-1}}
$$

$\mathrm{nf}_{\mathrm{k}}$ and $\mathrm{g}_{\mathrm{k}}$ are the noise figure and gain of section $\mathrm{k}$.

\section{EXPERIMENTAL RESULTS}

\section{A. SOA under test}

The structure of the fabricated SOA [6] is presented on Fig. 1. It has three sections biased by three independent currents. The device is a bulk InP/InGaAsP SOA, angled and anti-reflection coated, with a length of $700 \mu \mathrm{m}$.. The length of the contacts (cf. Fig. 1) is $236 \mu \mathrm{m}, 254 \mu \mathrm{m}$ and $210 \mu \mathrm{m}$. A $10 \mu \mathrm{m}$ slot gap provides a measured resistance between contacts of $300 \Omega$ (before FIB treatment). For manufacturing reasons, the current in each contact is limited to $90 \mathrm{~mA}$ and the total current to $150 \mathrm{~mA}$.

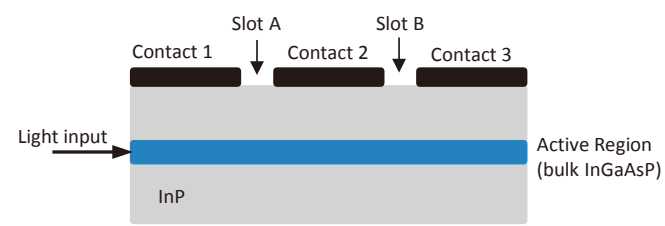

Fig. 1. Structure of the SOA under study presenting three electrodes biased independently.

Experimentally, the lowest noise figure is obtained when $90 \mathrm{~mA}$ is injected at contact $1,50 \mathrm{~mA}$ in contact 2 
and $10 \mathrm{~mA}$ at contact 3 . This distribution of current leads also to the lowest NF according to our simulation.
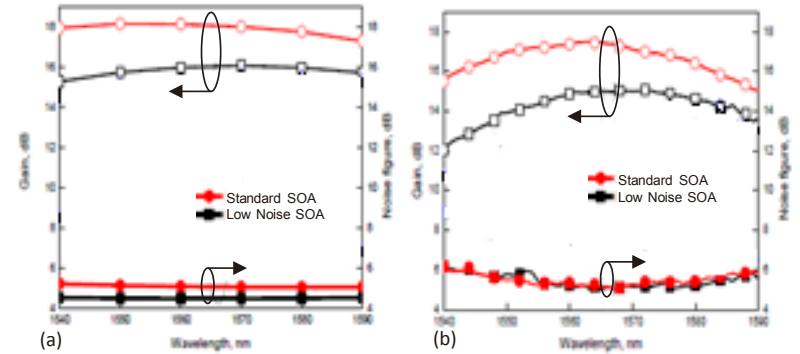

Fig. 2. Gain and noise figure as a function of wavelength from (a) simulation and (b) experiment.

Figure 2 shows an acceptable agreement on the order of magnitude for both noise figure and gain between the simulation and experimental results. The gain for the low noise $\mathrm{SOA}$ is lower by an average of $2 \mathrm{~dB}$ compared to a standard SOA. The NF is comparable between both SOAs, despite the simulated noise figure exhibiting a reduction of $0.5 \mathrm{~dB}$ between standard and low-noise configurations. One reason for this discrepancy is the limited resistance between contacts that does not stop carrier diffusion, which causes a change in the carrier distribution in the case of the low nf configuration making this distribution different from the optimal one.

In order to try to increase the inter-contact resistance by deeper etching of the slots between contacts, a focused ion beam (FIB) technique is used. A second SOA was used to test this approach. The dimensions of the three contacts are: $271 \mu \mathrm{m}$ for both sections 1 and 2 and 156.9 $\mu \mathrm{m}$ for section 3. Taking into account these lengths, the section currents are adapted. For a standard SOA, they are 59,58, $33 \mathrm{~mA}$, for section 1 to 3 ; for the low noise SOA configuration: 92, 43, $17 \mathrm{~mA}$. The inter-sectional resistances measured after FIB are $600 \Omega$ for slot A and $400 \Omega$ for slot B.
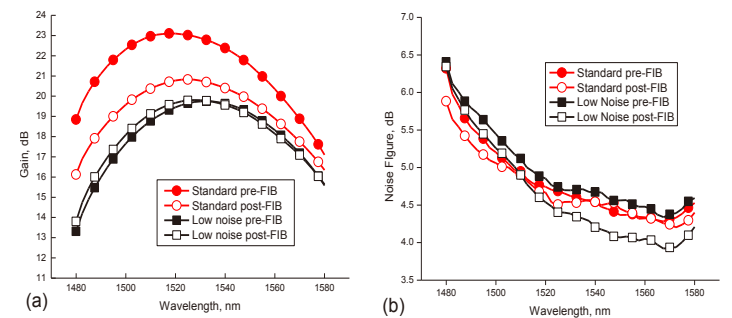

Fig. 3. (a) Gain and (b) noise figure as a function of wavelength for the fabricated SOA before and after etching. Red curves correspond to a standard SOA and the black curves to the low-noise configuration.

Figure 3 compares the gain and noise figure of the SOA before and after FIB etching. The gain difference between both SOAs has been reduced to less than $1 \mathrm{~dB}$ for wavelength greater than $1540 \mathrm{~nm}$. Due to the increase in resistance value between contacts, the NF difference between both SOAs is $0.45 \mathrm{~dB}$ at $1570 \mathrm{~nm}$. It can be noted that the NF for the low noise SOA has been reduced by $0.5 \mathrm{~dB}$ compared to the SOA before FIB. The minimum NF value obtained at $1570 \mathrm{~nm}$ is $3.8 \mathrm{~dB}$. This NF is extremely low, approaching the $3 \mathrm{~dB}$ limit, and is, to our knowledge, the lowest published for a bulk material SOA.
By controlling the carrier distribution in the SOA, it is therefore possible to reduce the noise-figure. This innovative design is compatible with the use of MQW, Qdash or Q-dot material, which would lead to further reduction of NF.

\section{Conclusions}

In this paper, the noise figure results of a multi-contact SOA with an engineered carrier distribution is investigated both from a numerical and experimental point of view. After FIB etching is performed to increase the resistance between contacts, the SOA exhibits a minimum noise figure of $3.8 \mathrm{~dB}$, which is to our knowledge, the lowest NF reported for a bulk device. The results presented are preliminary results and research is ongoing to improve the design and lower the NF toward the theoretical limit of $3 \mathrm{~dB}$.

\section{ACKNOWLEDGMENT}

This work was conducted under Enterprise Ireland, EU/ERDF (research project CFTD/2009/0303) and under the framework of the Irish Government's Programme for Research in Third Level Institutions Cycle 5, National Development Plan 2007-2013 with the assistance of the European Regional Development Fund.

\section{REFERENCES}

[1] Y. Liu, E. Tangdiongga,Z. Li, H. de Waardt, A.M. J. Koonen, G.D. Khoe, X. Shu, I. Bennion, and H.J.S. Dorren, "Error Free 320Gb/s All-Optical Wavelength Conversion using a Single Semiconductor Optical Amplifier”, J. Lightw. Technol., vol 25, pp. 103-108, 2007.

[2] G. Giulani and D. D'Alessandro, "Noise Analysis of Conventional and Gain-Clamped Semiconductor Optical Amplifiers", J. Ligh. Tech., vol 18, pp. 1256-1263, Sept. 2000

[3] M.J. Adams, J.V. Collins and I.D. Henning, 'Analysis of semiconductor laser optical amplifiers', Proc. Inst. Elec. Eng., vol 132, pp. 141-148, 1982

[4] T. Durhuus, B. Mikkelsen and K. Stubkjaer, "Detailled dynamic model for semiconductor optical amplifiers and their crosstalk and intermodulation distortion", J. Lightw. Technol., 1992, vol 10, pp. 1056-1065, 1992.

[5] M.J. Connelly, "Wideband semiconductor optical amplifier steady-state numerical model", IEEE J. Quantum Electron., vol 37, pp. 439-447, 2001.

[6] P.Landais and F. Surre, "A semiconductor optical amplifier with a reduced noise figure," UK patent GB0821602.0, Feb. 9, 2011 